\title{
CARACTERIZACIÓN DEL BARRIO ALEXIS ARGÜELLO, ESTELÍ, NICARAGUA.
}

\section{CHARACTERIZATION OF THE ALEXIS ARGÜELLO NEIGHBORHOOD, ESTELÍ, NICARAGUA.}

\author{
Luis María Dicovskiy Rioboo ${ }^{5}$ \\ Mariliana Videa Bustillo ${ }^{6}$ \\ Dominga Dalila González Cantarero ${ }^{7}$
}

RESUMEN: Con la finalidad de conocer la problemática y las necesidades de colaboración de la UNI sede Regional Norte en los tres sectores del Barrio Alexis Argüello, en Estelí, se aplicaron 361 encuestas casa a casa. Se encontró que casi todas las familias hay alguien asalariados, generalmente trabajador de fábrica o comercio, la mayoría de los niños en edad escolar asiste a la escuela y la mayoría de las familias dicen tener título de propiedad de su casa. Las casas tienen energía eléctrica y agua potable en su mayoría. Además de contar con acceso a televisión e incluso cable, en una buena parte de estas. Sin embargo, se encontró un grado de pobreza económica en la población, la generalidad de las casas tenía piso de tierra, paredes de madera y servicio sanitario por letrina. Pero el aspecto más sensible para el barrio era el mal estado de sus calles y drenajes. A la universidad le piden que apoye las gestiones del barrio y ayude en la formulación de proyectos.

PALABRAS CLAVE: socioeconómico, calles, drenajes, seguridad

ABSTRACT: In order to know the problems and the collaboration needs of the UNI North Regional with the Alexis Argüello neighborhood, in Estelí, 361 house-to-house surveys were applied. It was found that almost all families are salaried, most children of school age attend school and most families say they have title to their home. The houses have electricity and drinking water mostly. In addition to having access to television and even cable, in a good part of these. However, a degree of economic poverty was found in the population, the majority of the houses had a dirt floor, wooden walls and a sanitary service for a latrine. But the most sensitive aspect for the neighborhood was the poor condition of its streets and drains. The university is asked to support the efforts of the neighborhood and help in the formulation of projects.

KEYWORDS: socioeconomic, streets, drainages, security

\section{INTRODUCCIÓN}

\footnotetext{
${ }^{5}$ Docente UNI Sede Regional Norte, luis.dicovskiy@norte.uni.edu.ni

${ }^{6}$ Docente UNI Sede Regional Norte ingmarilianavidea@gmail.com

${ }^{7}$ Docente UNI Sede Regional Norte, dalicanta@gmail.com
} 
Este estudio se hizo con la finalidad de conocer la problemática y las necesidades de colaboración con la UNI sede Regional Norte, del Barrio Alexis Argüello, barrio aledaño a la universidad. Este barrio surgió de una toma de terrenos por parte de la población, en el año 2013, y está ubicado en el distrito I, costado sur oeste de la ciudad de Estelí. En la actualidad según datos del censo actualizado del Instituto de Formación Permanente (INSFOP) cuenta con 1,053 viviendas y 5,000 habitantes aproximadamente (Pérez Rugama \& Rizo Escorcia, 2015).

Las principales características socioeconómicas de las familias son que existen muchas mujeres que forman parte de una economía informal, la mayoría de las familias de este barrio son jóvenes y poseen bajos salarios familiares (Blandón Machado, 2016). Un artículo de la revista Confidencial comenta que "en el barrio Alexis Argüello las calles destrozadas y fangosas son una molestia" (Miranda Aburto, 2017). El barrio está dividido de este a Oeste en tres sectores, el I, II y III. El sector I es el que está más cerca de la UNI Norte.

\section{METODOLOGÍA}

En la última semana de octubre del 2016, docentes y estudiantes de la Sede UNI Norte hicieron 361 encuestas casa a casa en el Barrio Alexis Argüello. Estas encuestas, cuya mayoría de preguntas eran cerradas, se hicieron al azar en los tres sectores del barrio I, II y III, en días de semana y en horario laborable. De las casas encuestadas el $11 \%$ pertenecían al sector uno del barrio Alexis Arguello, el $14 \%$ al sector dos y otro $14 \%$ al sector tres, el $60 \%$ no identificó a que sector del barrio pertenecía. Ver Figura I, mapa del barrio.

Figura I: Mapa del Barrio Alexis Argüello

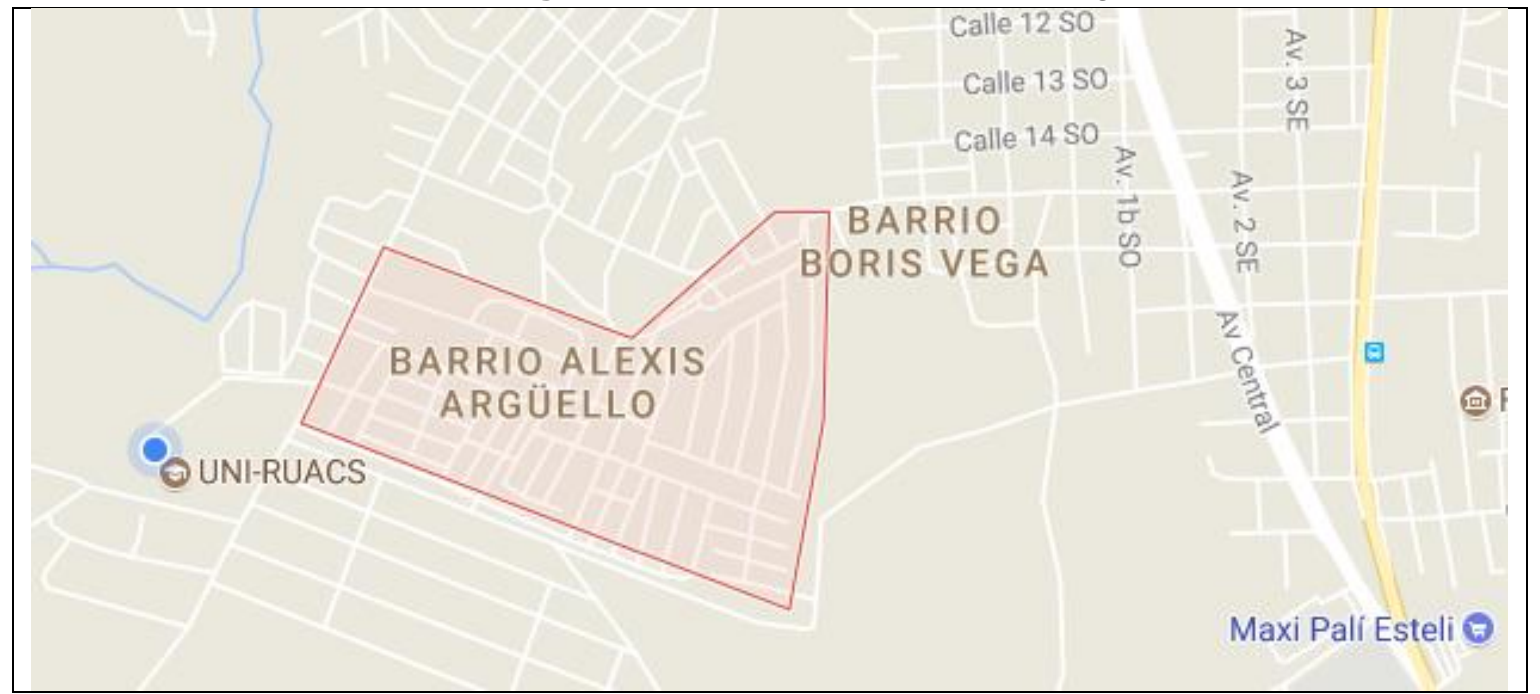

Fuente: Google Maps

\section{RESULTADOS Y DISCUSIÓN}

Datos Demográficos. El 17\% de las personas encuestadas manifestaron tener menos de 20 años cumplidos, un $28 \%$ tenían una edad entre 20 y 30 años, y un $50 \%$ más de 30 años cumplidos. La mayoría tenían una edad superior a los 20 años y pudieron responder de forma efectiva las preguntas de la encuesta. El $6.1 \%$ de los encuestados no manifestó su edad o no hay registro de la misma. 
La mayoría de las personas encuestadas fueron mujeres (69\%), se supone que esto se deba en parte a que generalmente quien trabaja en el hogar es el padre de familia, y es más fácil que en horas de la tarde se encuentre la madre en el hogar.

Datos Socioeconómicos. En cada hogar donde se aplicó la encuesta del barrio Alexis Argüello se encontró que habitan de 4 a 5 personas de promedio por casa, se contabilizaron de promedio entre 1 y 2 niños en edad escolar de 6 a 13 años en cada casa, de estos el $81 \%$ asiste a la escuela primaria.

Los habitantes del barrio tienen en promedio 5 años de vivir en este y en sus casas tienen entre 1 a 2 habitaciones para dormir, lo que indica que han acondicionado sus casas en este periodo de tiempo. El $89 \%$ de las familias dijeron tener título de propiedad de sus casas y el $76 \%$ manifestó no estar organizado. Sin embargo, se encontró que no hay diferencias significativas en los años y tener o no el título de propiedad, con un valor de $p<0.0001$ de una Prueba "t". El grupo que no posee título de propiedad tiene en promedio 3.48 años de vivir en el barrio, y el que, si lo posee, tiene 5.35 años.

En cada hogar hay de promedio entre 1 a 2 trabajadores activos, con un trabajo que proporciona el sustento del hogar. El $34 \%$ de las personas que habitan las casas son asalariados. Ellos principalmente trabajan en fábricas, cómo en el tabaco, en la construcción y en menor nivel en el comercio, ver tabla 1, Tipo de Trabajo.

Tabla 1: Tipo de trabajo

\begin{tabular}{crr}
\hline Tipo de Trabajo & Frecuencia & Porcentaje \\
\hline Fábrica & 98 & 27,1 \\
Comercio & 52 & 14,4 \\
Construcción & 40 & 11,1 \\
Domestico & 18 & 5,0 \\
Seguridad & 13 & 3,6 \\
\hline Otro & 124 & 34,3 \\
\hline Total & $\mathbf{3 6 1}$ & $\mathbf{1 0 0}$ \\
\hline
\end{tabular}

Fuente: Elaboración propia

Para conocer el estado de la salud de los habitantes del barrio, se les preguntó si actualmente había personas enfermas en el hogar, de 361 hogares encuestados solamente 93 manifestaron la presencia de enfermedades en el hogar, correspondiente al 25.8\%. Así mismo se les pregunto qué tipo de enfermedad es la que se estaba padeciendo y las respuestas se reflejan en la siguiente tabla del Tipo de enfermedad.

Los encuestados dieron respuestas numerosas al tipo de enfermedad que se estaba padeciendo en su familia en ese momento. Entre el 3 y $4 \%$ de las familias había gente con enfermedades semejantes al dengue y alrededor del $5 \%$ con problemas respiratorios o de gripe. 
En lo referente a animales domésticos casi la mitad de los hogares de este barrio tiene como animal doméstico a los perros, el animal más frecuente, y solo el 1\% tiene un cerdo en el hogar, el cual es un problema ambiental. Pocos indican que tienen otro tipo de animal doméstico que pueden ser pájaros, palomas o pericos,

Cuando se les preguntó a los habitantes del barrio si existía un riesgo ambiental en su casa. En más de la mitad de los casos dijeron que sí existía riesgo y dieron diversos tipos de este, entre los que se destacan: inundaciones debido a las lluvias, el $23.5 \%$ dijo que existía este peligro en el hogar, situación que se agrava por las calles que prestan limitaciones para el drenaje ya que no se cuenta con servicio de alcantarillado sanitario. Otro problema vinculado al anterior son los charcos que se forman en las calles, ya que son foco de enfermedades, con $13 \%$ de incidencia y la falta de aguas negras con un $2.8 \%$ de ocurrencia.

En cuanto al combustible que se usa en el hogar para cocinar, el $73 \%$ de los hogares utilizan gas, siendo el combustible más usado. Y el resto, $27 \%$ utilizan leña para esta labor.

En lo referente al manejo de las excretas, predomina el uso de letrina como servicio sanitario con un $80 \%$ de casas con este tipo de servicio, un $18 \%$ cuenta con inodoro y un $2 \%$ manifiesta no tener ninguno de los dos servicios sanitarios, ver gráfico de servicio sanitario.

En cuanto al manejo de la basura el $62 \%$ de las familias dicen utilizar el tren de aseo municipal, el resto no especifica que hace con la basura, lo que hace suponer que aquí hay un problema ambiental, ver gráfico de manejo de la basura.

Según tipo de vivienda, el $70 \%$ de las casas tienen piso de tierra, el $51 \%$ paredes de tabla y el $98 \%$ techo de zinc. Se observa que hay necesidad de mejorar las paredes y los pisos de las casas, los cuales son precarios.

Figura 2: Problemas prioritarios del Barrio

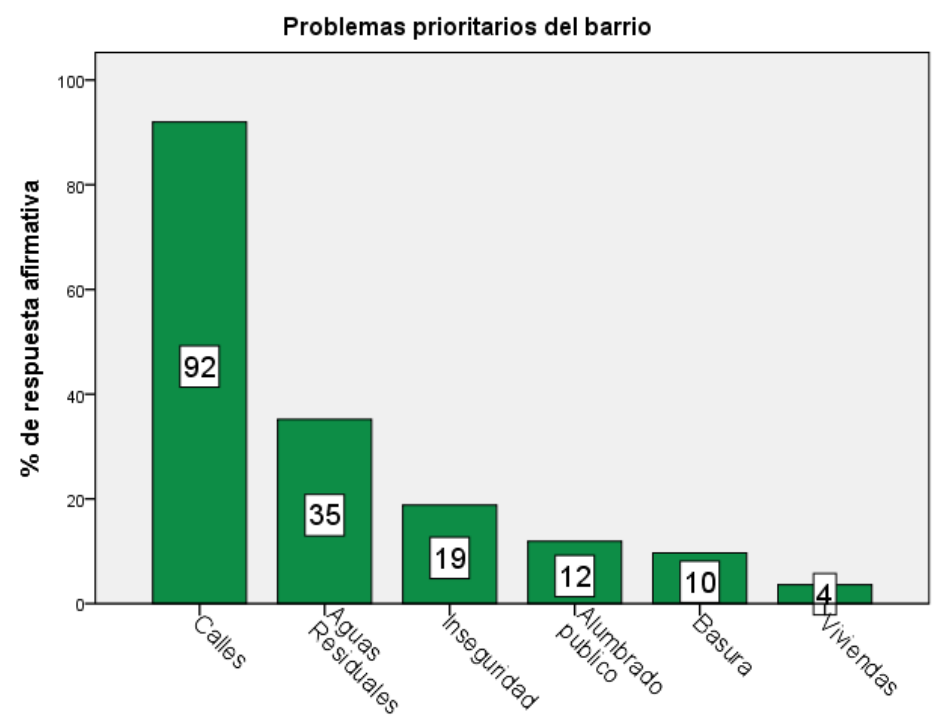

El Higo-Volumen 6. pp. 21-29/Diciembre 2016 
En cuanto a servicios disponibles, la mayoría de las casas tiene agua potable, $97 \%$ y electricidad, $97 \%$. En cuanto a comunicación en la mayoría de las casas hay teléfonos celulares, $91 \%$ y televisión, $89 \%$ y casi la mitad tiene TV por cable, $43 \%$.

Referente a los principales problemas que hay en el barrio, la gran mayoría de los encuestados mencionan el mal estado de las calles, esto se asocia también a problemas con el drenaje y la falta de aguas residuales. El problema de inseguridad pasa a un tercer lugar, ver figura II de problemas del barrio.

Sin embargo, la valoración de inseguridad es diferente según sector, valor de Chi Cuadrado de 0,0066 ( $p>0.05$ ). La inseguridad parece ser mayor en el Sector I, el más cercano a la UNI Norte. ver figura 3.

Figura 3: Gráfico Biplot, Inseguridad y Sector del Barrio

\section{Biplot de Sector e Inseguridad}

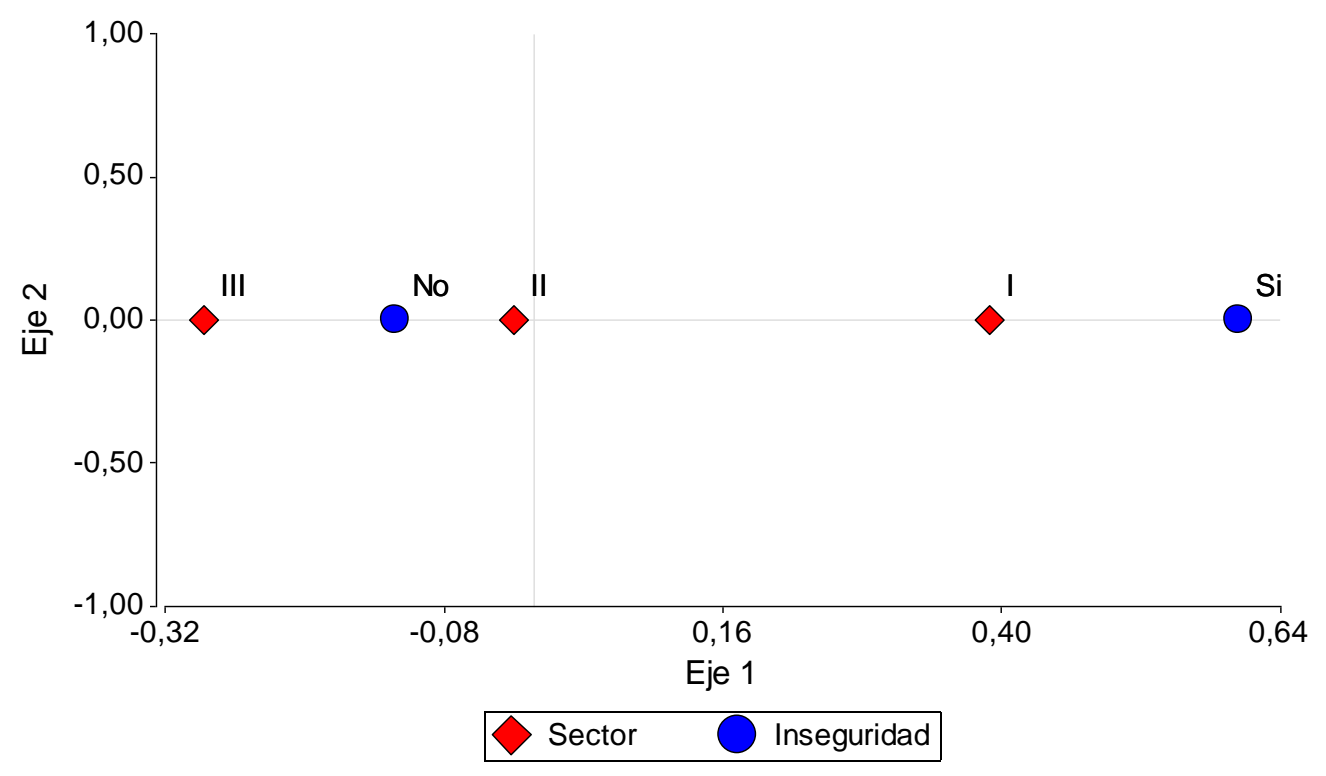

Ante la pregunta, qué es lo que hay que hacer para mejorar las condiciones del barrio surgieron ideas como: mejorar, calles, organización del barrio, elaborar proyectos comunales y acercar la policía al barrio, ver Figura 4 de Propuestas de Mejora. 
Figura 4: Propuestas de mejora

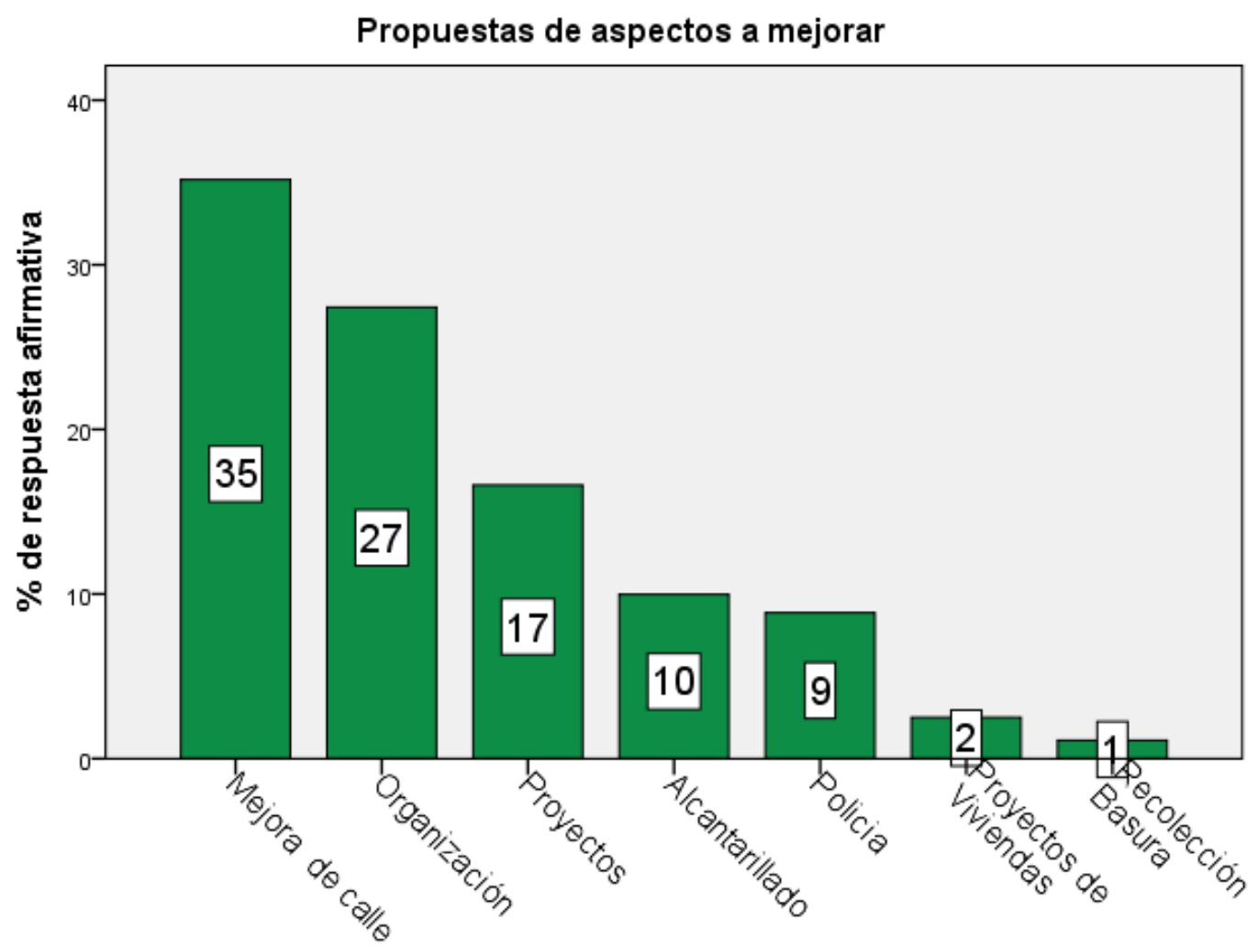

Colaboración con la UNI Norte. Ante la pregunta de "que esperan haga la UNI Norte por el barrio", las categorías de respuesta de mayor frecuencia fueron: acompañamientos con gestiones, 39\%, y también con proyectos de desarrollo, 37\%, ambos conceptos son complementarios y factibles de realizar cómo universidad,

Al analizar la relación la petición de apoyo a la universidad y el sector del barrio, se encontró que existe relación entre el sector y solicitud de gestiones por parte de la UNI Norte, específicamente el sector tres, con $49.02 \%$ pide este apoyo de la universidad, valor de Chi Cuadrado de esta pregunta fue de $0.0116(p<0.05)$. En la figura 6 se observan fotos del Sector I del barrio. 
Figura 5: Gráfico Biplot Solicitud a la UNI y Sector del Barrio Biplot en Análsis de Correspondencias

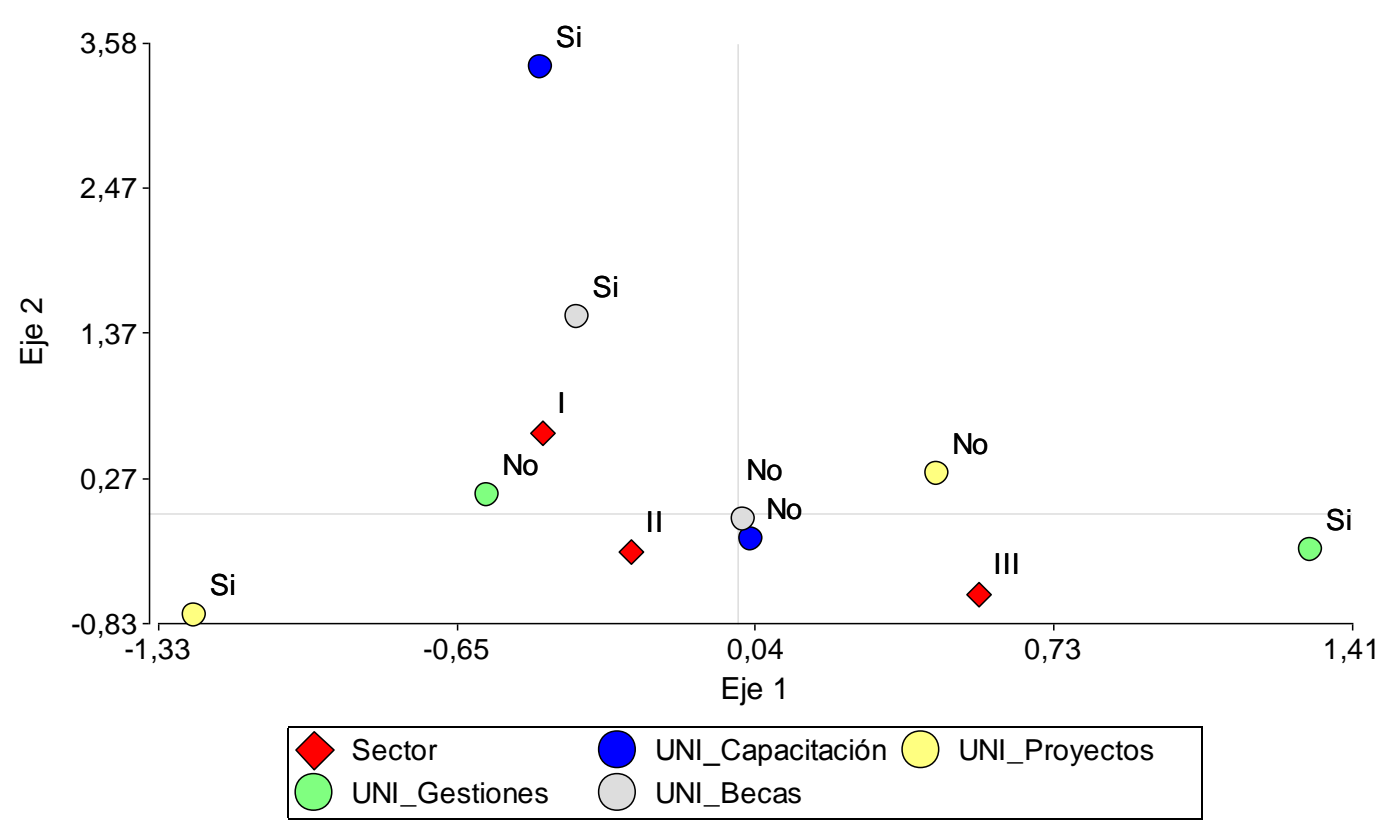

Figura 6: Fotos del Barrio Alexis Argüello, Sector I
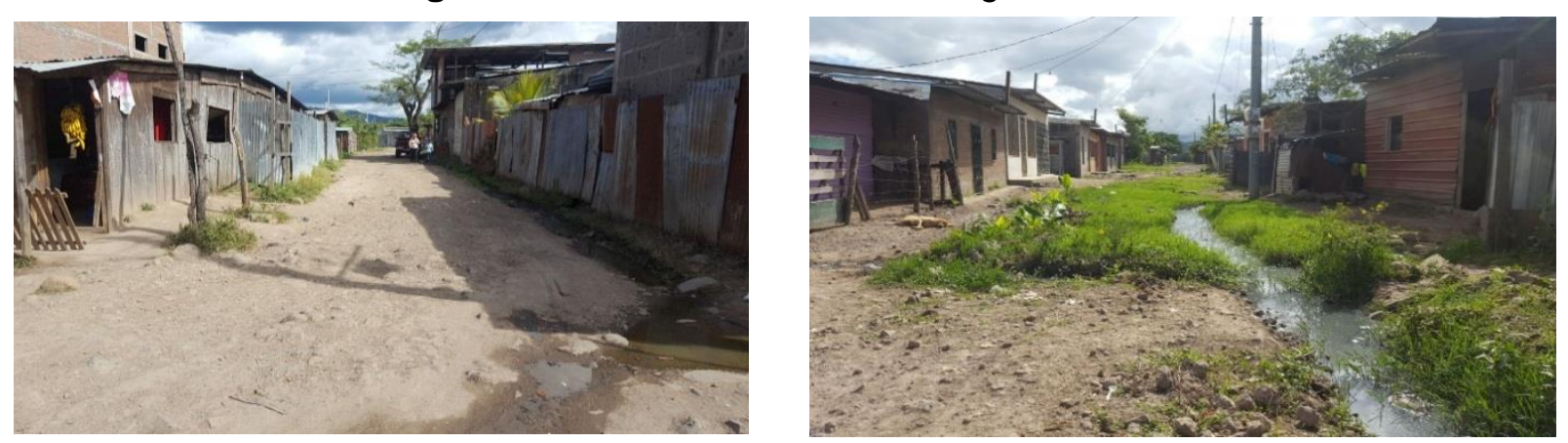

Fuente: Propia

\section{CONCLUSIONES}

En casi todas las familias hay alguien asalariado, la mayoría de los niños en edad escolar asiste a la escuela y casi todas las familias dicen tener título de propiedad de su casa. También se resalta como algo positivo, el hecho de que la mayoría de las casas tienen energía eléctrica y agua potable. Además de contar con acceso a televisión e incluso cable, en una buena parte de estas.

Como debilidades, un cuarto parte de las familias dijeron tener enfermos en las casas, siendo lo más común gripes o derivados del dengue. Pero el mayor problema que percibe el barrio, son las calles en mal estado, asociado esto con la falta de drenaje. Esta situación es en parte, 
producto del origen del barrio, que, al surgir de una toma, no tuvo ningún tipo diseño, ordenamiento o planificación de desarrollo. También es importante resaltar aspectos que indican un grado de pobreza económica en la población como son el hecho de que la mayoría de las casas tiene piso de tierra, paredes de madera y servicio sanitario por letrina.

\section{NOTACIÓN}

UNI: Universidad Nacional de Ingeniería.

UNI Norte: Sede Regional Norte de la Universidad Nacional de ingeniería, situada en Estelí, frente al barrio Alexis Argüello.

\section{REFERENCIAS}

Blandón Machado, Y. C. (2016). Situación socioeconómica de las familias del barrio Alexis Argüello en la ciudad de Estelí en el primer semestre del año 2016. Estelí: UNAN FAREM Estelí. Recuperado el 14 de Noviembre de 2017, de http://repositorio.unan.edu.ni/5117/1/17837.pdf

Miranda Aburto, W. (3 de Octubre de 2017). Un alcalde con "dos sombreros". El Confidencial. Recuperado el 14 de Noviembre de 2017, de https://confidencial.com.ni/alcalde-dos-sombreros/

Pérez Rugama, O. I., \& Rizo Escorcia, M. T. (2015). Factores que inciden positivamente para que los jóvenes en rehabilitación logren reinsertarse en la vida social, laboral y educativa, del Barrio Alexis Arguello de la Ciudad de Estelí. Estelí: UNAN FAREM Estelí.Obtenidode http://webcache.googleusercontent.com/search?q=cache:http://repositorio.unan.edu.ni/1960/1/1 6397.pdf

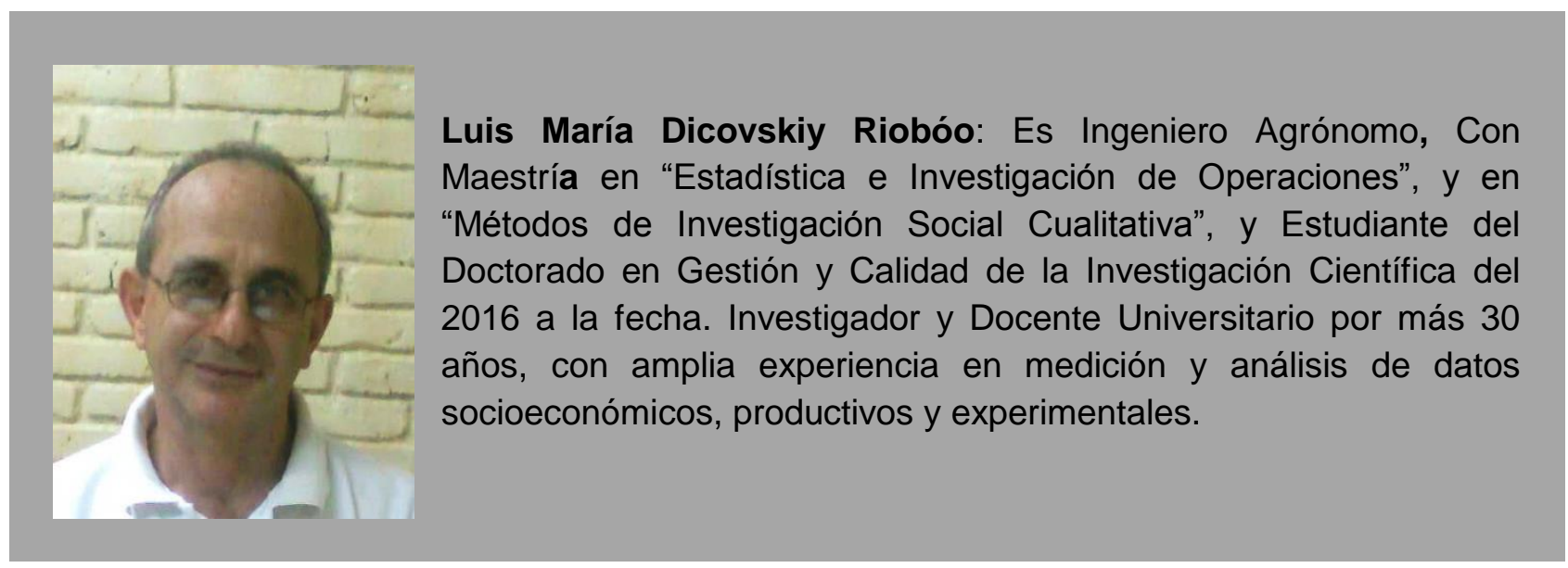



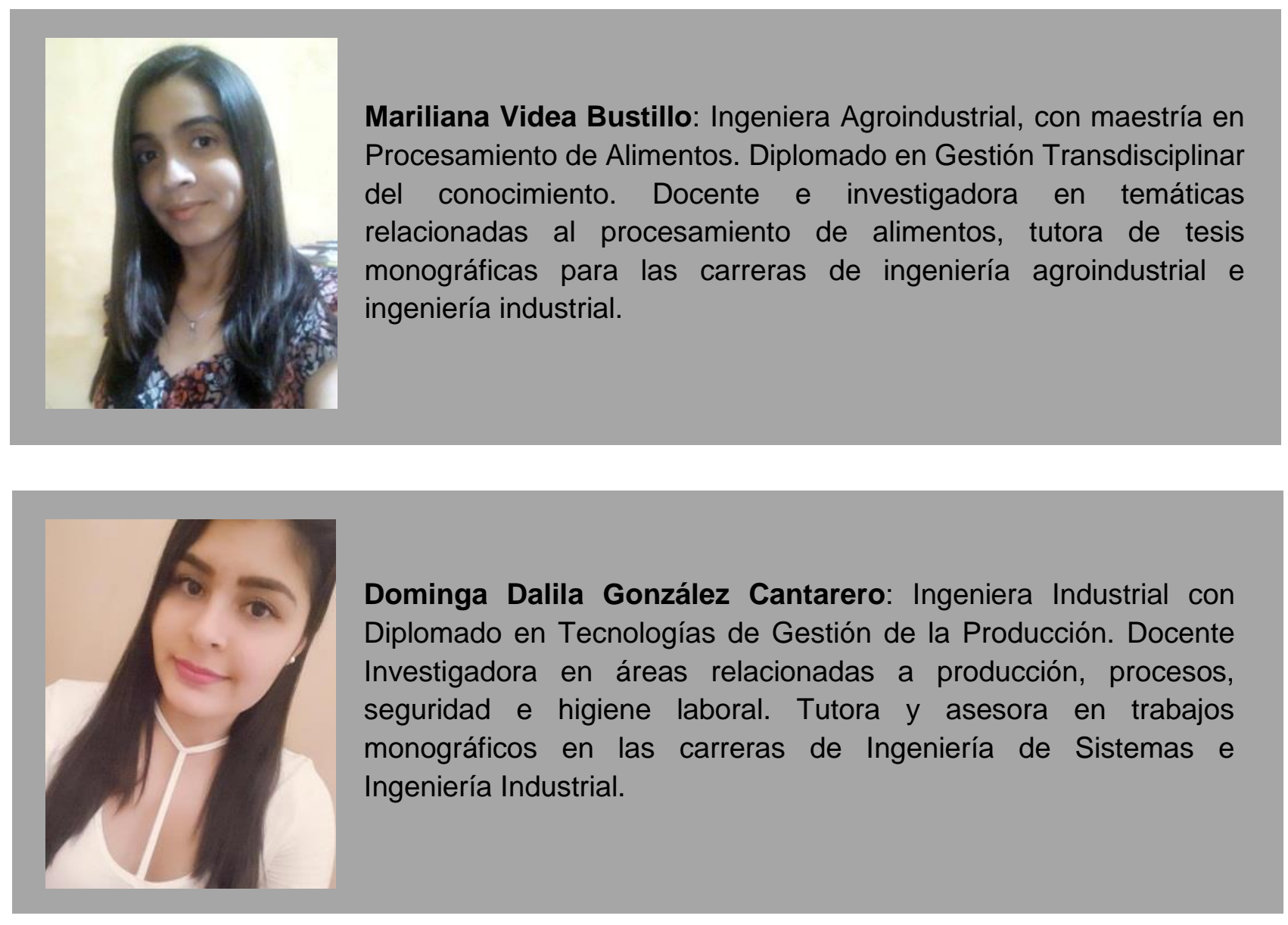